\title{
Up and Downlink Admission/Congestion Control and Maximal Load in Large Homogeneous CDMA Networks
}

François Baccelli — Bartłomiej Błaszczyszyn — Mohamed Kadhem Karray

\section{$\mathbf{N}^{\circ} 4954$}

Octobre 2003

THÈME 1 



\title{
Up and Downlink Admission/Congestion Control and Maximal Load in Large Homogeneous CDMA Networks
}

\author{
François Baccelli* , Bartłomiej Błaszczyszyn ${ }^{\dagger}$, Mohamed Kadhem Karray ${ }^{\ddagger}$ \\ Thème 1 -Réseaux et systèmes \\ Projet TREC
}

Rapport de recherche $\mathrm{n}^{\circ} 4954$-Octobre 2003 -27 pages

\begin{abstract}
This paper proposes scalable admission and congestion control schemes that allow each base station to decide independently of the others what set of voice users to serve and/or what bit rates to offer to elastic traffic users competing for bandwidth. These algorithms are primarily meant for large CDMA networks with a random but homogeneous user distribution. They take into account in an exact way the influence of geometry on the combination of inter-cell and intra-cell interferences as well as the existence of maximal power constraints of the base stations and users. We also study the load allowed by these schemes when the size of the network tends to infinity and the mean bit rate offered to elastic traffic users. By load, we mean here the number of voice users that each base station can serve.
\end{abstract}

Key-words: CDMA, power control, admission and congestion control, stochastic geometry, percell probability of rejection

\footnotetext{
* INRIA-ENS, 45 rue d'Ulm 75005, Paris, France, francois . baccelli@ens . fr

$\dagger$ INRIA-ENS, 45 rue d'Ulm 75005, Paris, France, Bartek. Blaszczyszyn@ens. fr

F France Télécom R\&D, 38/40 rue du Général Leclerc, 92794 Issy-Moulineaux France, mohamed.karray@rd.francetelecom.com
} 


\section{Contrôle d'Admission et de Congestion et Charge Maximale sur les Voies Montante et Descendante de Grands Réseaux CDMA Homogènes}

Résumé : Cet article propose des algorithmes de contrôle d'admission et de congestion permettant à chaque station de base de décider indépendamment des autres comment partager la bande passante, en déterminant les terminaux à débit constant (voix) à accepter et les débits à donner aux terminaux à débit élastique (données). Ces algorithmes sont conçus pour les grands réseaux CDMA ayant une distribution aléatoire mais homogène de terminaux. Ils prennent en compte de manière exacte l'influence de la géométrie sur les interférences intra et inter cellulaires ainsi que les limitations en puissance des stations de base et des terminaux. Nous étudions aussi le nombre des terminaux à débit fixe acceptés par le réseau lorsque la taille de ce dernier tend vers l'infini ainsi que le débit moyen offert aux terminaux à débit élastique.

Mots-clés : CDMA, contrôle de puissance, contrôle d'admission et de congestion, géometrie aléatoire, probabilité de rejet par cellule 


\section{Introduction}

This paper is concerned by the evaluation of the global up and down-link maximal load of CDMA networks. More precisely we analyze the maximal number of users that such a network can serve at a given bit-rate and/or the maximal bit-rates that such a network can provide to a given user population when taking into account

- the limitations of load due to inter-cell and own-cell interferences;

- maximal-power constraints.

This maximal load is evaluated and is used in order to define

- Admission control policies in the case of predefined user bit-rates (e.g., voice); i.e., schemes allowing one to decide whether a new user can be admitted or should be rejected as its admission could make the global power allocation problem unfeasible;

- Congestion control policies in the case of users with elastic bit-rates (e.g., data); i.e., schemes allowing one to determine the maximal fair user bit-rates that preserve the feasibility of the power control problem at any time, in function of the user population in all cells at this time.

The evaluation part relies on planar point processes and stochastic geometry. The model has several key components, the spatial location pattern of base stations (BS's), the spatial location pattern of users, the attenuation (path-loss) function and the policy of assignment of users to BS's, which are geometry-dependent, in addition to the non-geometric components such as orthogonality factors, pilot powers and external noise. We consider two specific models of BS locations: the hexagonal and the Poisson models, for which we show maximal load estimations.

We allow both patterns of locations to be countably infinite so as to address the scalability questions, and to check the ability of the proposed algorithms to continue to function well as the size of the network goes to infinity.

The basic assignment policy will be that where each mobile is served by the closest BS. It is basically equivalent to the optimal-SIR-choice scheme and to the honeycomb model in the classical hexagonal case.

This paper builds upon and complements a previous study in [1] which focuses on the downlink. The main novelties are the design of new algorithms for the uplink and the generalization of the protocols introduced in [1] allowing one to take to take the maximal power constraints.

The paper is organized as follows. We first give a brief survey of the literature in Section 2 and in Section 3 fix the notation and recall some very basic formulas concerning CDMA. In Section 4 we remind the formulations of the power control problems and give sufficient conditions for the existence of solutions. The new decentralized admission and congestion control algorithms based on these conditions are introduced in Section 5. The maximal load estimations are treated in Section 6. Numerical studies are gathered in Section 7. 


\section{Related work}

The problem of estimating the maximal load of CDMA networks has already been considered by several authors. Nettleton and Alavi [2] first considered the power allocation problem in the cellular spread spectrum context.

In Gilhousen et al [3], the problem was posed in the following way. Suppose Base Station number 1 (BS 1) emits at the total power $P_{1}$ in the presence of $K-1$ other BS's, which emit at power $P_{2}, \ldots, P_{K}$ respectively. How many users $N_{1}$ can then BS 1 accommodate assuming that the load of the network is only interference-limited and that each user has a required bit rate of $W$ ? The sufficient condition (and thus conservative load constraint) proposed in [3] reads

$$
\sum_{i=1}^{N_{1}} \xi_{i}\left(1+\sum_{k=2}^{K} \frac{\left(P_{k}\right)_{i}}{\left(P_{1}\right)_{i}}+\frac{\mathcal{N}}{\left(P_{1}\right)_{i}}\right) \leq 1 .
$$

In this formula, $\left(P_{k}\right)_{i}$ is the power received by user $i$ from BS $k$ and $\xi_{i}=\left(E_{b} / N_{0}\right)_{i} /(\pi W / R)$, where $\left(E_{b} / N_{0}\right)_{i}$ is the bit energy-to-noise density ratio of user $i ; R, \pi, \mathcal{N}$ are the bandwidth, the fraction of the total power devoted to the pilot signal and the external noise, respectively.

This simple condition allows for the determination of $N_{s}$ but it does not reflect a key feature, that in reality the total power emitted by the BS should depend on the number of users (and even on their locations), namely $P_{k}$ should be a function $P_{k}\left(N_{1}, \ldots, N_{K}\right)$.

In order to address this issue, Zander $[4,5]$ expresses the global power allocation problem by the multidimensional linear inequality

$$
\mathbb{Z} \mathbf{P} \leq \frac{1+\xi}{\xi} \mathbf{P}
$$

with unknown vector $\mathbf{P}$ of emitted powers; here one assumes the required signal-to-interference ratio $\xi$ (or equivalently the required user bit rate) to be given and one assumes the matrix $\mathbb{Z}$, the $i, k$ th entry of which gives the normalized path-losses between user $i$ and $\mathrm{BS} k$, to be given too. The main result is then that the power allocation is feasible if there exists a non-negative, finite solution to (2.2); the necessary and sufficient condition is that $\xi<1 /\left(\lambda^{*}-1\right)$, where $\lambda^{*}$ is the spectral radius of the (positive) matrix $\mathbb{Z}$. In order to simplify the problem, all same-cell channels are assumed to be completely orthogonal and the external noise is suppressed.

Foschini and Miljanic [6] and Hanly [7] introduced external noise to the model: Foschini considered a narrow-band cellular network and Hanly a two-cell spread spectrum network. On the basis of the previous works, Hanly extended the model in several articles. Hanly [8] extends this approach to the case with in-cell interference and external noise (essentially for the uplink). Using the block structure of $\mathbb{Z}$, he solves the problem in two steps: first the own-cell power allocation conditions are studied (microscopic view) and then the macroscopic view considers some aggregated cell-powers. He also interprets $\lambda^{*}$ as a measure of the traffic congestion in the network.

The evaluation of $\lambda^{*}$ can be done either from a centralized knowledge of the state of the network, which is non practical in large networks, or by channel probing as suggested in $\S$ VIII of [8] and described in [9]. When it exists, the minimal finite solution of inequality (2.2) can also be evaluated in a decentralized way (using Picard's iteration of operator $\mathbb{Z}$, cf. the discussion in $\S$ IX 
of [10]). However this does not provide decentralized admission or congestion control algorithms, namely scalable ways of controlling the network population or bit rates in such a way that the power allocation problem remains feasible, namely that $\lambda^{*}$ remains less than $1+1 / \xi$.

The approach of $[4,8]$ is continued in [1], where decentralized admission/congestion control protocols are proposed for the downlink, without maximal power constraints. These protocols are based on the simple mathematical fact that the maximal eigenvalue of any sub-stochastic matrix (matrix with nonnegative entries, whose row sums are less than 1) is less than 1. This approach, when applied to the downlink power allocation problem, takes a form similar to (2.1), with the received power $\left(P_{k}\right)_{i}$ replaced by path-loss/gain from BS $k$ to user $i$. Since path-loss basically depends on the geometry only and neither on the number of users served nor on the powers emitted, our version of Equation (2.1) no longer depends implicitly on $N_{k}$.

In the present paper, we will discuss extensions of the above approach that capture both down and up-link and take into account the maximal power constraints.

\section{Notation and Basic Relations}

We will use the following notation:

\subsection{Antenna Locations and Path Loss}

- $\left\{Y^{u}\right\}_{u}=\mathcal{N}_{B S}$ : locations of BS's; $u$ is the number (index) of BS and $Y^{u}$ denotes its location.

- $S_{u}$ : set of mobiles served by BS $u$.

- $\left\{X_{m}^{u}\right\}=\mathcal{N}_{M}^{u}$ : locations of mobiles served by BS $u$.

- $\left\{X_{m}\right\}_{m}=\mathcal{N}_{M}$ : locations of all mobiles; $m$ denotes the mobile located at $X_{m}$.

- $L(y, x)$ : path-loss of the signal on the path $y \rightarrow x$

- $l_{\downarrow m}^{u}=L\left(Y^{u}, X_{m}^{u}\right)$ : path-loss of the signal on the downlink $Y^{u} \rightarrow X_{m}^{u}$,

- $l_{\uparrow}^{u}=L\left(X_{m}^{u}, Y^{u}\right)$ : path-loss of the signal on the uplink $X_{m}^{u} \rightarrow Y^{u}$.

\subsection{Engineering parameters}

- $\alpha_{u}$ : downlink (DL) orthogonality factor in BS $u$; let

$$
\alpha_{u v}= \begin{cases}\alpha_{u} & \text { if } u=v \\ 1 & \text { if } u \neq v\end{cases}
$$


- $\xi_{m}^{u}$ : SINR threshold for user $X_{m}^{u} ; \xi_{\downarrow}^{u}, \xi_{\uparrow}^{u}$ if it is necessary to distinguish the DL and uplink (UL). Moreover, for each SINR $\xi$, we define a modified SINR $\xi^{\prime}$ by

$$
\xi_{\downarrow_{m}}^{\prime u}=\frac{\xi_{\downarrow_{m}}^{u}}{1+\alpha_{u} \xi_{\downarrow_{m}}^{u}}, \quad \xi_{\uparrow_{m}}^{u}=\frac{\xi_{\uparrow_{m}}^{u}}{1+\xi_{\uparrow_{m}^{u}}^{u}}
$$

- $P_{\downarrow_{m}}^{u}$ : power of the dedicated channel $u \rightarrow m$,

- $P_{\uparrow}^{u}$ : power transmitted by mobile $m \rightarrow u$,

- $\tilde{P}^{u}$ : maximal total power of BS $u$ (for the load estimates we use also $\bar{P}_{\downarrow}$ to denote the average maximal power that does not depend on the BS index),

- $P^{\prime} u$ : total power of the common channels $(\mathrm{CCH})[11]$,

- $P^{u}=P^{\prime} u+\sum_{m} P_{\downarrow_{m}}^{u}$ : total power transmitted by BS $u$,

- $\tilde{P}_{m}^{u}$ : maximal power of mobile $m \in S_{u}$ (for the load estimates we use also $\bar{P}_{\uparrow}$ to denote the average maximal power that does not depend on the mobile index),

- $N$ : external noise; $N^{u}, N_{m}^{u}$, respectively, for the noise at BS $u$ and mobile $m \in S_{u}$,

- $R, R_{\downarrow m}^{u}, R_{\uparrow m}^{u}$ bit-rates; Note that the theoretical maximal bit-rate of the Gaussian channel is related to the $\operatorname{SINR} \xi$ by

$$
R=B \log (1+\xi),
$$

where $B$ is the bandwidth. In practice, the following bit-rate is implemented

$$
R=\frac{\xi W}{\left(E_{0} / I_{0}\right)},
$$

where $\left(E_{0} / I_{0}\right)$ bit-energy-to-noise-ratio density, $W$ is the chip-rate.

\section{Up and Downlink Power Control with Power Constraints}

In this section we describe the power control $\left({ }^{1}\right.$ ) problems with power constraints (so called feasibility problems) and give some sufficient conditions, called feasibility conditions, for the existence of solutions. These conditions are the basis for admission and congestion control algorithms proposed in the next section.

\footnotetext{
${ }^{1}$ We use equivalently power control and power allocation.
} 


\subsection{Feasibility of Power Control with Power Constraints}

\subsubsection{Downlink}

We will say that the (downlink) power allocation with power limitations is feasible if there exist nonnegative powers $P_{\downarrow}^{u}$ for all base stations $u$ and mobiles $m$, which satisfy the following two conditions:

i. signal to interference and noise ratio at each mobile is larger than the threshold $\xi_{\downarrow} u$; i.e.,

$$
\frac{P_{\downarrow_{m}}^{u} / l_{\downarrow_{m}}^{u}}{N_{m}^{u}+\sum_{v} \alpha_{u v}\left(P^{\prime} v+\sum_{n \in S_{v}} P_{\downarrow_{n}}^{v}\right) / l_{\downarrow_{m}}^{v}} \geq \xi_{\downarrow_{m}}^{u},
$$

for all $u$ and $m \in S_{u}$.

ii. the total power transmitted by each base station is not larger than its given limit $\sum_{m \in S_{u}} P_{\downarrow_{m}}^{u}+$ $P^{\prime} u \leq \tilde{P}^{u}$, for all $u$.

We will say that the (downlink) power allocation (without power limitations) is feasible if there exist nonnegative powers $P_{\downarrow_{m}}^{u}$ such that condition i) is satisfied.

\subsubsection{Uplink}

We will say that the (uplink) power control with power constraints is feasible if there exist nonnegative powers $P_{\uparrow}^{u}$ such that the following two conditions are satisfied:

i. signal to interference and noise ratio at each BS is larger than the threshold $\xi_{\uparrow_{m}^{u}}^{u}$; i.e.,

$$
\frac{P_{\uparrow_{m}^{u}}^{u} / l_{\uparrow_{m}}^{u}}{N^{u}+\sum_{v} \sum_{n \in S_{v}} P_{\uparrow_{n}^{v}}^{v} / l_{\uparrow_{n}^{u}}^{u}} \geq \xi_{\uparrow_{m}^{\prime}}^{u}
$$

for all $u, m \in S_{u}$.

ii. the power transmitted by each mobile is not larger than its given limit $P_{\uparrow}^{u} \leq \tilde{P}_{m}^{u}$, for all $u$ and $m \in S_{u}$.

We will say that the (uplink) power control (without power constraints) is feasible if there exist nonnegative powers $P_{\uparrow}^{u}$ such that condition i) is satisfied.

\subsection{Feasibility Conditions}

The following results hold (see the discussion in Section 4.3 and proofs in Section A.1). 


\subsubsection{Downlink}

DPAFC (Downlink Power Allocation Feasibility Condition): If for each BS $u$

$$
\sum_{m \in S_{u}} \sum_{v} \frac{\alpha_{u v} \xi_{\downarrow_{m}}^{u} l_{\downarrow_{m}}^{u}}{l_{\downarrow}^{v}}<1
$$

then the downlink power control without power limitations is feasible.

E-DPAFC (for Extended DPAFC): If for each BS $u$

$$
\sum_{m \in S_{u}}\left(N_{m}^{u}+\sum_{v} \alpha_{u v} \tilde{P}^{v} / l_{\downarrow_{m}}^{v}\right) l_{\downarrow_{m}}^{u} \xi_{\downarrow_{m}}^{u} \leq \tilde{P}^{u}-P^{\prime u}
$$

then the downlink power control with power limitations is feasible.

\subsubsection{Uplink}

UPAFC (Uplink Power Allocation Feasibility Condition): If for each BS $u$

$$
\sum_{m \in S_{u}} \sum_{v} \frac{\xi_{\uparrow_{m}}^{u} l_{m}^{u}}{l_{\uparrow m}^{v}}<1
$$

then the uplink power control without power limitations is feasible.

UBC \& ULC If for some collection of reals $\theta_{u} \in[0,1)$ the following two conditions are satisfied UBC (Uplink Budget Condition)

$$
\theta_{u} \leq 1-N^{u} \sup _{m \in S_{u}} \frac{\xi_{\uparrow_{m}^{\prime}}^{u} l_{\uparrow}^{u}}{\tilde{P}_{m}^{u}}
$$

for all $u$, and

\section{ULC (Uplink Link Condition)}

$$
\sum_{v} \sum_{n \in S_{v}} \frac{l_{\uparrow_{n}}^{v} \xi_{n}^{v} N_{n}^{v} N^{v}}{l_{n}^{u}\left(1-\theta_{v}\right)} \leq \frac{\theta_{u} N^{u}}{1-\theta_{u}}
$$

then the uplink power control problem with power limitations is feasible. 
E-UPAFC If for some collection of real numbers $\gamma_{u v} \geq 0$, such that $\gamma_{u}=\sum_{v} \gamma_{u v}<\infty$, the following two conditions are satisfied for all $u$

$$
\left(N^{u}+\gamma_{u}\right) \sup _{m \in S_{u}} \frac{\xi_{\uparrow_{m}^{\prime}}^{u} l_{m}^{u}}{\tilde{P}_{m}^{u}} \leq 1
$$

and

$$
\frac{\gamma_{u}+N^{u}}{\gamma_{v u}} \sum_{m \in S_{u}} \frac{\xi_{\uparrow_{m}^{\prime}}^{u} l_{m}^{u}}{l_{\uparrow_{m}^{v}}^{v}} \leq 1 \quad \text { for all } v,
$$

then the uplink power control problem with power limitations is feasible.

\subsection{Discussion of the Conditions}

\subsubsection{Decentralization}

We will say that a power control (or power allocation) condition is decentralized if it is of the form: For each BS u condition $\mathcal{C}_{u}$ holds, where $\mathcal{C}_{u}$ depends on the locations and parameters of the users $\left\{m \in S_{u}\right\}$ served by BS $u$ and, possibly, on the locations and parameters of all other BS's, but $\mathcal{C}_{u}$ does not depend on numbers, locations and parameters of $\left\{n \in S_{v}\right\}$ for $v \neq u$.

Note that our conditions DPAFC, E-DPAFC, UPAFC and E-UPAFC are decentralized in the sense of the above definition.

The interest in decentralized conditions stems from the fact that an admission or congestion control algorithm based on decentralized conditions is scalable when the size of the network (number of base stations) increases.

\subsubsection{Sufficiency}

All our conditions are sufficient, but not necessary ones. This means that the respective algorithms or maximal load estimates will be conservative. However, loosely speaking our conditions DPAFC, E-DPAFC, UPAFC and UBC \& ULC, E-UPAFC are almost necessary for a symmetric network with similar traffic for all BS's. Moreover the conditions E-DPAFC and UBC \& ULC are asymptotically equivalent to DPAFC and UPAFC, respectively, for large maximal power constraints. Below we explain the above statements.

It is known (see e.g., [12]) that the necessary and sufficient conditions for the feasibility of the downlink and uplink power allocation problems, both without power constraints, can be expressed in terms of the spectral radii of the matrices of the so-called normalized path-losses: $\mathbb{A}=\left(a_{u v}\right)$, $a_{u v}=\alpha_{u v} \sum_{m \in S_{u}} \xi_{\downarrow}^{u} l_{\downarrow_{m}}^{u} / l_{\downarrow_{m}}^{v}$ for the DL and $\mathbb{B}=\left(b_{u v}\right), b_{u v}=\sum_{n \in S_{v}} \xi_{\uparrow_{n}}^{v} l_{\uparrow_{n}}^{v} / l_{\uparrow}^{u}$ for the UL. (Note that the conditions based on the evaluation or estimation of the spectral radii of $\mathbb{A}, \mathbb{B}$ are not decentralized). Our conditions DPAFC and UPAFC say that the matrices, respectively, $\mathbb{A}$ and $\mathbb{B}$ are substochastic. More precisely, DPAFC says that all sums in lines of $\mathbb{A}$ are less than 1 , whereas UPAFC says that all sums in columns of $\mathbb{B}$ are less than 1 . The more lines (respectively columns) of these matrices are similar to each other, the less is the "space between" our sufficient conditions and the necessary ones (in particular, note that for a matrix with identical all lines (columns), the sums 
in lines (columns) are less than 1 if and only if the spectral radius is less than 1.) This means that our conditions DPAFC and UPAFC should be almost necessary for a symmetric network with random but homogeneous traffic. This claim is the object of ongoing experimental verification.

Concerning the non-necessity of conditions E-DPAFC and UBC \& ULC, which take into account power limitations, note first that letting $\tilde{P}^{u} \rightarrow \infty$ uniformly for all $u$, in the condition E-DPAFC, we get DPAFC. Similarly, assuming (for simplicity) $\theta_{u}=\theta$ and $N^{u}=N$ and letting $\tilde{P}_{m}^{u} \rightarrow \infty$ uniformly for all $u, m$ in the conditions UBC \& ULC, we get a condition stating that matrix $\mathbb{B}$ is substochastic (sums in lines less than 1). Thus we can say the conditions E-DPAFC and UBC \& ULC are asymptotically equivalent to DPAFC and UPAFC, respectively, for large maximal power constraints.

In order to see why for a given finite $\tilde{P}^{u}$ and $\tilde{P}_{m}^{u}$ the conditions E-DPAFC and UBC \& ULC are not necessary, note that all solutions (powers) of the power control problem 4.1.1i form a cone (whose intersection with the positive orthant might be empty). The E-DPAFC requires that some specific vector of powers, chosen such that the total power emitted by each BS is equal to its maximal total power, lies in this cone. It might in general be the case that the cone of solutions intersects the positive orthant but does not contain our specific solution. However, loosely speaking, this is more likely in a non-symmetric situation, e.g., when the BS are unequally loaded. Similar reasoning applies for the uplink power control problem 4.1.2i, with the specific solution on which conditions UBC \& ULC and E-UPAFC are based, being $N^{u} /\left(1-\theta_{u}\right)$ and $N^{u} \gamma_{u}$, respectively. All this means again that the conditions UBC \&ULC and E-UPAFC should be almost necessary for a symmetric network with similar traffic for all BS's.

\subsubsection{The choice of $\gamma_{u v}$ in E-DPAFC}

The condition ULC is not decentralized because it sets the threshold for the total interference received in each cell, and it is only the interference emitted by each cell that can be controlled locally. Thus, in order to guarantee the required level of the interference received in a decentralized manner, we have a priori to "reserve" some allowed levels of interference between each pair of cells. This is done by the choice of constants $\gamma_{u v}$. It seems reasonable to take these constants proportional to the mean interference; i.e., to the mean value of $\sum_{m \in S_{u}}[\ldots]$ in (4.7).

\subsubsection{Uplink implies downlink}

Note that our conditions DPAFC and UPAFC coincide if the path-loss does not depend on the direction of the communication (i.e., when $l_{\downarrow_{m}}^{u} \equiv l_{\uparrow m}^{u}$ ) provided orthogonality factors $\alpha_{u v}=1$, in the case when the SINR targets are the same for uplink and downlink. Moreover, under the same conditions, the normalized path-losses matrices $\mathbb{A}$ and $\mathbb{B}$ are identical up to transposition, making if-and-only-if conditions for both links equivalent. (A similar result for narrowband systems was given in [13].)

Since typically $\alpha_{u u}=\alpha_{u}<1$ thus UPAFC implies DPAFC and it is the uplink that forms the bottleneck for the power allocation problem when there are no power constraints and when the traffic (bit-rate) is symmetric between the uplink and the downlink. 


\subsubsection{UBC \& ULC - coverage and load}

The choice of the parameter $\theta$ in conditions (4.4) and (4.5) is related to the classical coverage/load tradeoff. Small $\theta$ allows for more distant users in UBC, and thus gives larger coverage but reduces load in UBC. Large (close to 1) $\theta$ gives larger load in ULC but rejects remote users in UBC. We will address the problem of the choice of $\theta$ in Section 6.3.1.

\section{Protocols Based on the Decentralized Conditions}

The decentralized conditions of the previous section allow us to define admission control policies in the case of predefined customer bit rates (e.g., voice); i.e., schemes allowing one to decide whether a new customer can be admitted or should be rejected as its admission could make the power allocation and/or problem unfeasible, and congestion control policies in the case of customers with elastic bit rates (e.g., data); i.e., schemes allowing one to determine the maximal fair customer bit rates that preserve the feasibility of the power allocation and/or control problem at any time, in function of the customer population in all cells at this time.

\subsection{Admission Control}

Assume the bit rates of all users (or equivalently all $\xi_{\downarrow_{m}}^{\prime u}, \xi_{\uparrow_{m}}^{\prime u}$ parameters) to be specified. The admission control problem can then be posed as follows: for a given mobile population $\left\{m \in S_{u}\right\}$, for all BS $u$, check whether the (downlink) power allocation and the (uplink) power control with power constraints (defined in Section 4.1) are feasible. Here we describe (conservative) procedures built from the (sufficient) decentralized conditions given in Section 4.2,

\subsubsection{Downlink}

Define the user's $m \in S_{u}$ downlink-power-control load with respect to BS $u$

$$
f_{\downarrow_{m}}^{u}=\left(N_{m}^{u}+\sum_{v} \frac{\alpha_{u v} \tilde{P}^{v}}{l_{\downarrow_{m}^{v}}^{v}}\right) \frac{l_{\downarrow_{m}}^{u} \xi_{\downarrow_{m}}^{u}}{\tilde{P}^{u}} .
$$

Note that condition (4.1) is equivalent to

$$
\sum_{m \in S_{u}} f_{\downarrow}^{u}<1,
$$

with $\tilde{P}^{v}=\tilde{P}^{u}=\infty$ in (5.1), whereas condition (4.2) is equivalent to

$$
\sum_{m \in S_{u}} f_{\downarrow m}^{u} \leq 1-\frac{P^{\prime} u}{\tilde{P}^{u}} .
$$

This leads to the following algorithm. 
(Extended) Downlink Admission Control Protocol ((E-)DACP): Each BS checks periodically whether condition (5.2) (or (5.3)) is satisfied and, if not, enforces it by reducing the population $\left\{m: m \in S_{u}\right\}$ of its mobiles to some subset s.t. the inequality holds with the reduced population. When a new mobile user applies to some BS, the BS accepts it if the respective condition is satisfied with this additional user and rejects it otherwise.

Note that the application of DACP by all the BS's guarantees the global feasibility of the downlink power control problem 4.1.1i without power constraints, whereas the E-DACP guarantees in addition that the solution of the power allocation problem satisfies the maximal-power constraints 4.1.1ii. Moreover, it was shown in [1] that under DPAFC condition $P_{\downarrow m}^{u}=\tilde{P}^{u} f_{\downarrow m}^{u}$ for $m \in S_{u}$ are individual solutions of the problem 4.1.1i.

\subsubsection{Uplink}

Define the user's $m \in S_{u}$ uplink-power-control load with respect to BS $v$

$$
f_{\uparrow m}^{u v}=\frac{\xi_{\uparrow m}^{\prime u} l_{\uparrow m}^{u}}{l_{\uparrow m}^{v}}
$$

and the aggregated uplink-power-control load

$$
f_{\uparrow m}^{u}=\sum_{v} f_{\uparrow m}^{u v} .
$$

Note that the condition (4.3) is equivalent to

$$
\sum_{m \in S_{u}} f_{\uparrow m}^{u}<1,
$$

whereas (4.7) is equivalent to

$$
\sum_{m \in S_{u}} f_{\uparrow m}^{u v} \leq \frac{\gamma_{u v}}{\gamma_{u}+N^{u}}, \quad \text { for all } v .
$$

Note also that (4.6) is satisfied iff

$$
\frac{\xi_{\uparrow}^{\prime u} l_{\uparrow m}^{u}}{\tilde{P}_{m}^{u}} \leq \frac{1}{N^{u}+\gamma_{u}}
$$

for all $m \in S_{u}$. The above formulations lead to the following algorithms.

Uplink Admission Control Protocol (UACP): Each BS checks periodically whether condition (5.6) is satisfied and, if not, enforces it by reducing the population $\left\{m: m \in S_{u}\right\}$ of its mobiles to some subset s.t. the inequality holds with the reduced population. When a new mobile user applies to some BS, the BS accepts it if the condition is satisfied with this additional user and rejects it otherwise.

Extended Uplink Admission Control Protocol (E-UACP): Each BS first reduces the population of its mobiles $\left\{m: m \in S_{u}\right\}$ to those which satisfy condition (5.8). Moreover, each BS checks 
periodically whether conditions (5.7) are satisfied by the reduced population and, if not, enforces it by further reducing the population to some subset s.t. the inequalities hold with the further-reduced population. When a new mobile user applies to some BS, the BS accepts it if it satisfies (5.8) and if the conditions (5.7) are satisfied with this additional user; the mobile is rejected if one of the above conditions is violated.

Note that the application of the UACP by all the BS's guarantees the global feasibility of the uplink power control problem 4.1.2i without power constraints, whereas the application of the EUACP by all the BS's guarantees the global feasibility of the uplink power control problem 4.1.2i with power constraints 4.1.2ii.

We see that the problem of power constraints in the uplink is more tricky than in the downlink because it requires individual control of the interference emitted toward each BS (cf. (5.7)). However, note that if in a symmetric case $\gamma_{u v}=\gamma_{v u}$ we replace the collection of inequalities (5.7) by one condition adding the inequalities up; i.e.,

$$
\sum_{m \in S_{u}} f_{\uparrow}^{u} \leq \theta_{u},
$$

taking $\theta_{u}=\gamma_{u} /\left(\gamma_{u}+N^{u}\right)$ and consequently rewriting (5.8)

$$
\frac{\xi_{\uparrow_{m}^{u}}^{\prime u} l_{m}^{u}}{\tilde{P}_{m}^{u}} \leq \frac{1-\theta_{u}}{N^{u}}
$$

we can propose the following heuristic algorithm.

Simplified Extended Uplink Admission Control Protocol (SE-UACP): Each BS first reduces the population of its mobiles $\left\{m: m \in S_{u}\right\}$ to those which satisfy condition (5.10). Moreover, each BS checks periodically whether condition (5.9) is satisfied by the reduced population and, if not, enforces it by further reducing the population to some subset s.t. the inequality holds with the further-reduced population. When a new mobile user applies to some BS, the BS accepts it if it satisfies (5.10) and if the condition (5.9) is satisfied with this additional user; the mobile is rejected it if one of the above conditions is violated.

Note that the application of the SE-UACP by all the BS's only roughly ensures the global feasibility of the uplink power control problem 4.1.1i with power constraints 4.1.1ii.

\subsection{Congestion Control}

In this section, we do not assume the bit rates of users (or equivalently the $\xi_{\downarrow}^{\prime u}, \xi_{\uparrow_{m}}^{\text {u }}$ parameters) to be specified. We are interested in a scheme for elastic traffic, namely for traffic which can accommodate bit rate variations. We consider the case with no admission control, where an increase of the number of users in a cell is just coped with via a reduction of the bit rates of the users of this cell, like in TCP where the increase of the number of competitors eventually results in a decreased bit rate for all, and where no user is ever rejected. We will look for a fair scheme in the downlink and the so-called max-min fair scheme in the uplink. This means all users in a given cell are supposed to have exactly the same $\xi_{\downarrow}^{u}$ in the downlink, whereas for the uplink, $\xi_{\uparrow_{m}^{u}}^{u}\left(m \in S_{u}\right)$ results from the classical water-filling policy. 


\subsubsection{Downlink}

Assume $\xi_{\downarrow_{m}}^{\prime u}=\xi_{\downarrow}^{\prime u}$, for all $m \in S_{u}$. Bearing in mind the relation (3.1) note that (4.2) is equivalent to

$$
\xi_{\downarrow}^{u} \leq \frac{1}{\max \left(0, \frac{\sum_{m \in S_{u}}\left(N_{m}^{u}+\sum_{v} \alpha_{u v} \tilde{P}^{v} / l_{\downarrow}^{v}\right) l_{\downarrow}^{u}}{\tilde{P}^{u}-P^{\prime u}}-\alpha_{u}\right)} .
$$

This leads to the following algorithm.

Downlink Congestion Control Protocol (DCCP): Each BS periodically allocates to all mobiles in its cell the fair downlink rate $R_{\downarrow}^{u}=R$ given by (3.3) with SINR $\xi=\xi_{\downarrow}^{u}$ satisfying equality in (5.11). This fair rate is also updated at any time when a customer joins or leaves the cell.

\subsubsection{Uplink}

We first consider the condition E-UPAFC. Looking for a fair assignment of bit-rates (or equivalently $\xi_{\downarrow_{m}}^{u}$ or $\xi_{\downarrow_{m}}^{u}$ ) in the presence of the individual constraints (4.6) requires application of the so called max-min fair policy (or water-filling policy; see [14]). Roughly speaking, it aims at allocating as much load as they can accept to users experiencing difficult conditions, and an equal share to others. Formally, denote by

$$
\tilde{\xi}_{m}^{u}=\frac{\tilde{P}_{m}^{u}}{l_{\uparrow_{m}^{u}}^{u}\left(N^{u}+\gamma_{u}\right)}
$$

the maximal modified-SIR reachable by the mobile $m \in S_{u}$; define the equal-share level of the modified SIR by

$$
\xi_{\uparrow}^{\prime u}=\sup \left\{\xi: \sup _{v} \frac{\gamma_{u}+N^{u}}{\gamma_{v u}} \sum_{m \in S_{u}} \min \left(\xi, \tilde{\xi}_{m}^{u}\right) \frac{l_{\uparrow_{m}^{u}}^{u}}{l_{\uparrow_{m}^{v}}^{v}} \leq 1\right\} .
$$

and the individual modified SINR's by

$$
\xi_{\uparrow_{m}^{\prime}}^{u}=\min \left(\tilde{\xi}_{m}^{u}, \xi_{\uparrow}^{\prime u}\right) .
$$

This leads to the following algorithm.

Uplink Congestion Control Protocol (UCCP): Each BS periodically allocates to each mobile in its cell the max-min fair uplink rate $R_{\uparrow_{m}}^{u}=R$ given by (3.3) with SINR $\xi=\xi_{\uparrow_{m}^{\prime}}^{\prime u} /\left(1-\xi_{\uparrow_{m}}^{\prime u}\right)$ where $\xi_{\uparrow}^{\prime u}$ is given by (5.14). This fair rate is also updated at any time when a customer joins or leaves the cell.

Note that similarly to admission control, we can implement a simplified (heuristic) uplink congestion control based on conditions (5.9) and (5.10). For this it suffices to replace the formulas (5.12) and (5.13), respectively by the following ones

$$
\tilde{\xi}_{m}^{u}=\frac{\tilde{P}_{m}^{u}\left(1-\theta_{u}\right)}{l_{m}^{u} N^{u}}
$$

and

$$
\xi_{\uparrow}^{\prime u}=\sup \left\{\xi: \sum_{m \in S_{u}} \min \left(\xi, \tilde{\xi}_{m}^{u}\right) \sum_{v} \frac{l_{\uparrow_{m}^{u}}^{u}}{l_{\uparrow_{m}^{v}}^{v}} \leq \theta_{u}\right\} .
$$




\section{Maximal Load Estimations based on the Decentralized Con- ditions}

The decentralized conditions of Section 4.2 also provide conservative bounds for the maximal load of the network. We will consider two extreme and complementary architectures: the infinite Hexagonal model that represents large perfectly structured networks and the infinite, homogeneous Poisson model that takes into account irregularities of a large real networks in a statistical way. In both models we assume stationary Poisson distribution of users, and also assume that each is served by its nearest BS.

Fix a network architecture and its parameters, in particular the spatial density of its BS's.

The maximal admission load estimation for fixed bit rate traffic aims at finding the maximal density of users such that the decentralized conditions of Section 4.2 hold with sufficiently high probability.

The maximal throughput estimation for elastic traffic aims at finding the maximal fair bit-rates such that the decentralized conditions of Section 4.2 hold with sufficiently high probability.

\subsection{From Hexagonal to Poisson Model}

Denote by $\lambda_{B S}$ the mean number of BS's per $\mathrm{km}^{2}$. Each BS serves users in its cell defined as the set of locations in the plane which are closer to that BS than to any other BS. It is convenient to relate $\lambda_{B S}$ to the radius $R$ of the (virtual) disc whose area is equal to that of the area (mean area, in the Poisson case) of the cell, by the formula

$$
\lambda_{B S}=1 /\left(\pi R^{2}\right)
$$

Bearing this definition in mind, we will sometimes call $R$ the radius of the cell.

\subsubsection{Hexagonal Model (Hex)}

In the hexagonal model, the radius $R$ is related to the the distance $\Delta$ between two adjacent BS's by $\Delta^{2}=2 \pi R^{2} / \sqrt{3}$. The BS's are located on the grid denoted on the complex plane by $\left\{Y^{u}: Y^{u}=\right.$ $\left.\Delta\left(u_{1}+u_{2} e^{i \pi / 3}\right), u=\left(u_{1}, u_{2}\right) \in\{0, \pm 1, \ldots\}^{2}\right\}$. The cell-pattern in this model is sometimes called honeycomb.

\subsubsection{Poisson Model (PV)}

In the Poisson case $\left\{Y^{u}\right\}$ constitutes the Poisson process on the plane, with intensity $\lambda_{B S}$. The cell pattern in this model are called Poisson-Voronoi tessellation. Note that in the Poisson-Voronoi model BS-locations as well as cells are random. We always assume that the Poisson process of BS's is independent of all other random elements considered in the sequel.

In both models we assume a homogeneous Poisson process of users $\left\{X_{m}\right\}$, with intensity $\lambda_{M}$; thus $\lambda_{M}$ is the mean number of users per $\mathrm{km}^{2}$. In both models, we denote by $V^{u}$ the Voronoi cell of BS $Y^{u}$. BS $Y^{u}$ thus serves mobiles $\left\{m \in S_{u}\right\}=\left\{X_{m} \in V^{u}\right\}$. 
We model path-loss on distance $r$ by

$$
L(r)=(K r)^{\eta}
$$

where $\eta>2$ is the so-called path-loss exponent and $K>0$ is a multiplicative constant.

Other characteristics, like powers $\tilde{P}^{u}, \tilde{P}_{m}$, SINR's $\xi_{\downarrow_{m}^{u}}^{u}, \xi_{\uparrow_{m}^{u}}^{u}$ etc, are assumed in both models to be independent, identically distributed random variables. It is reasonable to assume that common channel powers $P^{\prime} u$ are given by independent, identically distributed fractions $P^{\prime} u / \tilde{P}^{u}$.

\subsection{Mean Value Approach}

A first estimate of maximal load that provides explicit formulas consists in studying the conditions of Section 4.2 "in mean". In order to present the results, it is convenient to adopt the following notation. Let $\bar{M}=\lambda_{M} / \lambda_{B S}$ be the mean number of users per BS. Moreover denote by $\bar{N}=\mathrm{E} N$, $\bar{\xi}_{\downarrow}^{\prime}=\mathrm{E} \xi_{\downarrow}^{\prime}, \bar{\xi}_{\uparrow}^{\prime}=\mathrm{E} \xi_{\uparrow}^{\prime}, \bar{P}_{\downarrow}=\mathrm{E} \tilde{P}^{u}, \bar{P}_{\uparrow}=\mathrm{E} \tilde{P}_{m}$ the respective means of the noise, modified SINR's and maximal powers. Let $\bar{\pi}=\mathrm{E}\left[P^{\prime} / \tilde{P}\right]$ be the mean fraction of the maximal power devoted to the common channels.

\subsubsection{Downlink}

Taking expectation on both sides of (4.2), we get the following relation between the mean number of users per cell and the radius $R$ of the cell in the context of power allocation with power constraints.

$$
\bar{M} \leq \frac{1-\bar{\pi}}{\bar{\xi}_{\downarrow}^{\prime}\left(\alpha+\bar{f}+L(R) \bar{N} \bar{g} / \bar{P}_{\downarrow}\right)}
$$

where

$$
\bar{f}= \begin{cases}\bar{f}_{\mathrm{PV}}=2 /(\eta-2) & \text { for the PV model } \\ \bar{f}_{\mathrm{Hex}} \approx 0.9365 /(\eta-2) & \text { for the Hex model }\end{cases}
$$

(the approximation for the Hex model is a least square fit of some more precise function by the linear function of $1 /(\eta-2)$, for $\eta \in[2.2,5])$;

$$
\bar{g}= \begin{cases}\bar{g}_{\mathrm{PV}}=\Gamma(1+\eta / 2) & \text { for the PV model } \\ \bar{g}_{\mathrm{Hex}} \approx(1+\eta / 2)^{-1} & \text { for the Hex model }\end{cases}
$$

Moreover, letting $\bar{P}_{\downarrow} \rightarrow \infty$, and $\bar{\pi} \rightarrow 0$, in (6.2) we get the mean version of the condition DPAFC (4.1).

\subsubsection{Uplink}

Assuming $\theta_{u}=\theta$ and comparing the expectation of the left-hand side of (4.5) to some lower bound (Jensen inequality) of the expectation of the right-hand-side of (4.4), we get the following relation between the mean number of users $\bar{M}$ per cell and the radius $R$ of the cell (in the context of power 
control with power constraints and of constant ${\xi_{\uparrow}^{\prime}}_{m}^{u} / \tilde{P}_{m}^{u} \equiv \bar{\xi}_{\uparrow}^{\prime} / \bar{P}_{\uparrow}$, i.e., when neglecting the variation of $\xi_{\uparrow}^{\prime}$ and $\tilde{P}$ with respect to the variation of the path-loss):

$$
\bar{M} \leq \frac{1 / \bar{\xi}_{\uparrow}^{\prime}-\bar{N} L(R) \bar{h}(\bar{M}) / \bar{P}_{\uparrow}}{1+\bar{f}},
$$

where $\bar{f}$ is given by (6.3) and the function $\bar{h}(s)$ for PV and Hex model, respectively, is given by

$$
\bar{h}(s)=\left\{\begin{array}{l}
\bar{h}_{\mathrm{PV}}(s)=\int_{0}^{\infty} 1-e^{-s e^{-z^{2 / \eta}}} d z \\
\bar{h}_{\mathrm{Hex}}(s) \approx \int_{0}^{1} 1-e^{-s\left(1-z^{2 / \eta}\right)} d z .
\end{array}\right.
$$

Moreover, letting $\bar{P}_{\uparrow} \rightarrow \infty$ in (6.5) we get the mean version of the condition UPAFC (4.3).

Replacing $\sup _{m \in S_{u}}$ over the random set of users in (4.4) by the sup over the entire cell in Hex model, we get a simplified, but a bit more restrictive "mean load with full coverage":

$$
\bar{M} \leq \frac{1 / \bar{\xi}_{\uparrow}^{\prime}-L(R) \bar{N} / \bar{P}_{\uparrow}}{1+\bar{f}} .
$$

\subsection{Probability of Rejection}

Another and more accurate load estimation consists in looking for the maximal density of users such that our decentralized admission control protocols admit all users with a given (high) probability (we concentrate here on admission control). The problem can be formalized as follows. Fix a typical BS, say no 0, and let the "CONDITION" be any of the conditions \{DPAFC, E-DPAFC, UPAFC, UBC\&UBC, E-UPAFC, (5.9)\&(5.10)\}.

Maximal load at a given probability of rejection: For a given density of $B S \lambda_{B S}>0$ (equivalently, cell radius $R<\infty)$ and CPR (Cell Probability of Rejection) $\epsilon>0$, let $\lambda_{M}^{\epsilon}=\lambda_{M}^{\epsilon}\left(\lambda_{B S}\right)$ be the maximal density of users (equivalently, the mean number $\bar{M}=\bar{M}^{\epsilon}(R)$ of users per cell) such that

$$
\operatorname{Pr}(\text { “CONDITION” holds for typical BS }) \geq 1-\epsilon .
$$

This requires estimates for the distribution functions of the sums $\sum_{m \in S_{0}} f_{m}$, where $f_{m}$ is the load associated to the user $m$ in the "CONDITION". Note also that (4.6) requires ways of estimating the distribution function of the random variable $\sup _{m \in S_{0}} \xi_{\uparrow m}^{\prime 0} l_{\uparrow}^{0} / \tilde{P}_{m}^{0}$.

In the next subsection we briefly review the main ideas for estimating these distributions and hence CPR: simulation and analytical bounds or approximations.

Before this, we will comment on the choice of the parameter $\theta$ in UBC\&ULC.

\subsubsection{Coverage/Load tradeoff}

Note that in the uplink we have two competing conditions (4.4) and (4.6) related to, respectively, coverage and load (see Section 4.3.5). In this context, two error-probabilities are usually given: 
$\epsilon_{\mathrm{cov}}>0$ and $\epsilon_{\mathrm{cap}}>0$, with typically $\epsilon_{\mathrm{cov}}<\epsilon_{\mathrm{cap}}$. The maximal load can then be defined as the maximal density of users such that $U B C$ and $U L C$ holds with probabilities $1-\epsilon_{\text {cov }}$ and $1-\epsilon_{\text {cap }}$, respectively. Since $\epsilon_{\mathrm{cov}}$ is typically very small, the following simplified solution, which guarantees full coverage in the Hex model, looks satisfactory: First choose $\theta$ that satisfies condition (4.4) with sup taken over the whole cell (and not over the random set of users in the cell). Then use this value of $\theta$ to calculate the maximal load via (4.5) at the given $\epsilon_{\text {cap }}$.

\subsubsection{Techniques for CPR estimation}

Let $f_{m}, m \in S_{0}$ be the loads of users brought to the typical BS (say no 0) according to the given CONDITION. The point is to estimate the probability of events of the form:

$$
\mathcal{E}(z)=\left\{\sum_{m \in S_{0}} f_{m} \geq z\right\}
$$

for $z \geq 0$.

Complete simulation We choose a discrete set of test intensities (of mobiles) $\lambda_{0}<\lambda_{1}<\ldots<\lambda_{k}$ and simulate $k$ independent patterns of Poisson point processes $\mathcal{N}_{i}(i=0, \ldots, k)$ with respective intensities $\lambda_{0}$ and $\Delta_{i}=\lambda_{i}-\lambda_{i-1}$ in the cell $V^{0}$ of BS 0 generated by a given pattern $\mathcal{N}_{B S}$. Let $F_{i}(z)=\mathbf{I}(\mathcal{E}(z))$ be the indicator that the event (6.9) holds for the population of mobiles $\mathcal{N}_{M}=\sum_{j=0}^{i} \mathcal{N}_{j}$. Obviously $\mathrm{E}\left[F_{i}\right]=\operatorname{Pr}(\mathcal{E}(z))$ at $\lambda_{M}=\lambda_{i}$ and $F_{i}$ is increasing in $i$. The same holds for $F_{i}^{(n)}=1 / n \sum_{u=1}^{n} F_{i, u}$, where $\left(F_{i, u}, i=0, \ldots, k\right), u=1, \ldots, n$ are independent copies of $\left(F_{i}, i=0, \ldots, k\right)$. In addition, $F_{i}^{(n)}$ converges a.s. to $\operatorname{Pr}(\mathcal{E}(z))$ at $\lambda_{M}=\lambda_{i}$ as $n \rightarrow \infty$.

Chebychev's inequality This requires some ways of estimating (upper-bounding) of the variance $\operatorname{Var}\left[\sum_{m \in S_{0}} f_{m}\right]$. If it is available, then

$\operatorname{Pr}(\mathcal{E}(z)) \leq \operatorname{Var}\left[\sum_{m \in S_{0}} f_{m}\right] /\left(z-\mathrm{E}\left[\sum_{m \in S_{0}} f_{m}\right]\right)^{2}$.

Gaussian approximation If an estimator (upper bound) of the variance $\operatorname{Var}\left[\sum_{m \in S_{0}} f_{m}\right]$ is available, then

$$
\operatorname{Pr}(\mathcal{E}(z)) \approx Q\left(\frac{z-\mathrm{E}\left[\sum_{m \in S_{0}} f_{m}\right]}{\sqrt{\operatorname{Var}\left[\sum_{m \in S_{0}} f_{m}\right]}}\right),
$$

where $Q(z)=1 / \sqrt{2 \pi} \int_{z}^{\infty} e^{-t^{2} / 2} d t$ is the Gaussian tail distribution function.

\subsubsection{Formulas}

We now give explicit formulas for exact values or bounds of the probabilities, expectations, variances and Laplace transforms required in techniques mentioned in previous section. We concentrate on the condition E-DPAFC for the downlink with its associated load $f_{\downarrow}^{0}$ given by (5.1) and on the 
conditions UPAFC and (5.9), with their load $f_{\uparrow}^{0}$ given by (5.5). For the user-maximal power constraints, we give approximations of the tail distribution function of the sup in (4.4) and (4.6). For simplicity, we assume that all the users and BS characteristics but their locations are deterministic (their variations are negligible with respect to the variations due to locations of antennas).

\section{Probabilities}

$$
\operatorname{Pr}\left(\sup _{m \in S_{0}} \frac{\xi_{\uparrow} l_{\uparrow_{m}^{0}}^{0}}{\tilde{P}}>z\right) \leq 1-\exp \left[-\bar{M} \phi\left(\left(\frac{z \tilde{P}}{\xi_{\uparrow}^{\prime} L(R)}\right)^{2 / \eta}\right)\right]
$$

where

$$
\phi= \begin{cases}\phi_{\mathrm{PV}}=e^{-r} & \text { for PV model } \\ \phi_{\mathrm{Hex}} \approx \max (0,1-r) & \text { for Hex model }\end{cases}
$$

and for the Hex model we have equality in (6.10).

\section{Expectations}

$$
\begin{aligned}
\mathrm{E}\left[\sum_{m \in S_{0}} f_{\downarrow}^{0}\right] & =\bar{M} \bar{\xi}_{\downarrow}^{\prime}\left(\alpha+\bar{f}+L(R) \bar{N} \bar{g} / \bar{P}_{\downarrow}\right) \\
\mathrm{E}\left[\sum_{m \in S_{0}} f_{\uparrow}^{0}{ }_{m}^{0}\right] & =\bar{M} \bar{\xi}_{\uparrow}^{\prime}(1+\bar{f}),
\end{aligned}
$$

where $\bar{f}, \bar{g}$ are given by (6.3), (6.4), respectively.

Variances We concentrate on the Hex model only. We have

$$
\begin{aligned}
& \operatorname{Var}\left[\sum_{m \in S_{0}} f_{\downarrow m}^{0}\right]=\bar{M} \bar{\xi}_{\downarrow}^{2}\left(\bar{N}^{2} L^{2}(R) \bar{g}(2 \eta) / \bar{P}_{\downarrow}^{2}+\alpha^{2}+\overline{f^{2}}+2\left(\alpha \bar{f}+N L(R)(\alpha \bar{g}+\overline{l f}) / \bar{P}_{\downarrow}\right)\right) \\
& \operatorname{Var}\left[\sum_{m \in S_{0}} f_{\uparrow}^{0}\right]=\bar{M} \bar{\xi}_{\uparrow}^{\prime 2}\left(\overline{f^{2}}+1+2 \bar{f}\right)
\end{aligned}
$$

where $\bar{f}, \bar{g}$ are given by (6.3), (6.4), $\bar{g}(2 \eta)$ denotes $\bar{g}$ calculated at doubled path-loss exponent and

$$
\overline{f^{2}}={\overline{f^{2}}}_{\mathrm{Hex}} \approx \frac{0.2343}{(\eta-2)}+\frac{1.2907}{(\eta-2)^{2}}
$$

and

$$
\overline{l f}=\overline{l f}_{\mathrm{Hex}} \approx \frac{0.6362}{\eta-2} ;
$$

these approximations are appropriate least square fits for $\eta \in[2.5,5]$. We remark also that corresponding (exact) values for the PV model are $\bar{f}_{\mathrm{PV}}=8 /(\eta-2)^{2}+1 /(\eta-1)$ and $\overline{l f}_{\mathrm{PV}}=$ $\Gamma(2+\eta / 2) / 2$, but the formula for the variance requires some positive correcting term due to the randomness of the cell size. 


\subsubsection{Load for the Hex model}

Using the Gaussian approximation we get the following explicit formulas for the mean number of users per cell in the Hexagonal model at a given per-cell probability $\epsilon$ of rejection in E-DPAFC

$$
\bar{M} \leq \bar{M}_{\downarrow}-\frac{\left(Q^{-1}(\epsilon)\right)^{2} \overline{X_{\downarrow}^{2}}}{2 \bar{X}_{\downarrow}^{2}}\left(\sqrt{\frac{4(1-\bar{\pi}) \bar{X}_{\downarrow}}{\bar{\xi}_{\downarrow}^{\prime} \overline{X_{\downarrow}^{2}}}+1}-1\right),
$$

where $\bar{M}_{\downarrow}$ is the upper bound for $\bar{M}$ given by the mean model (i.e., the right-hand-side of (6.2)) and

$$
\begin{aligned}
\bar{X}_{\downarrow} & =\alpha+\bar{f}_{\mathrm{Hex}}+L(R) \bar{N} \bar{g}_{\mathrm{Hex}} / \bar{P}_{\downarrow} \\
\bar{X}_{\downarrow}^{2} & =\bar{N}^{2} L^{2}(R) \bar{g}_{\mathrm{Hex}}(2 \eta) / \bar{P}_{\downarrow}^{2}+\alpha^{2}+\bar{f}_{\mathrm{Hex}}+2\left(\alpha \bar{f}_{\mathrm{Hex}}+N L(R)\left(\alpha \bar{g}_{\mathrm{Hex}}+\overline{l f}_{\mathrm{Hex}}\right) / \bar{P}_{\downarrow}[]\right),
\end{aligned}
$$

Similarly for the uplink (considering SE-UACP with full coverage)

$$
\bar{M} \leq \bar{M}_{\uparrow}-\frac{\left(Q^{-1}(\epsilon)\right)^{2} \overline{X_{\uparrow}^{2}}}{2 \bar{X}_{\uparrow}^{2}}\left(\sqrt{\frac{4\left(1 / \bar{\xi}_{\uparrow}^{\prime}-L(R) \bar{N} / \bar{P}_{\uparrow}\right) \bar{X}_{\uparrow}}{\bar{X}_{\uparrow}^{2}}+1}-1\right)
$$

where $\bar{M}_{\uparrow}$ is the upper bound for $\bar{M}$ given by the mean model (i.e., the right-hand-side of (6.7) and

$$
\begin{aligned}
\bar{X}_{\uparrow} & =1+\bar{f}_{\mathrm{Hex}} \\
\overline{X_{\uparrow}^{2}} & =1+\bar{f}_{\mathrm{Hex}}^{2}+2 \bar{f}_{\mathrm{Hex}} .
\end{aligned}
$$

\section{Numerical Results}

\subsection{Model Specifi cation}

We will study the maximal load estimations for the models with different size of the typical cell, parametrized by the distance $R$ between adjacent BS's. The following values are fixed for the study.

Path Loss $\eta=3.38, K=8667$.

Physical layer parameters $\quad \alpha=0.4, \xi_{\downarrow}=\xi_{\uparrow}=-16 \mathrm{~dB}, N^{u}=-105 \mathrm{dBm}, N_{m}=-103 \mathrm{dBm}$ (external noise at the BS and user, respectively), $\tilde{P}^{u}=52 \mathrm{dBm}, \tilde{P}_{m}=33 \mathrm{dBm}$ (maximal powers of BS and mobile, respectively, including antenna gains and losses), $P^{\prime}=42.73 \mathrm{dBm}$. (The above values correspond to the UMTS system [11].)

Mean Factors For the specific value $\eta=3.38$ we get the following values of the mean factors: $\bar{g}_{\mathrm{PV}}=1.5325, \bar{g}_{\mathrm{Hex}}=0.3717, \bar{g}_{\mathrm{Hex}}(2 \eta)=0.2283, \bar{f}_{\mathrm{PV}}=1.4493, \bar{f}_{\mathrm{Hex}}=0.6564, \bar{f}^{2}{ }_{\mathrm{Hex}}=0.8703$, $\overline{l f}_{\mathrm{Hex}}=0.4394$ (see Section 6.3.3 and Appendix A.2). 


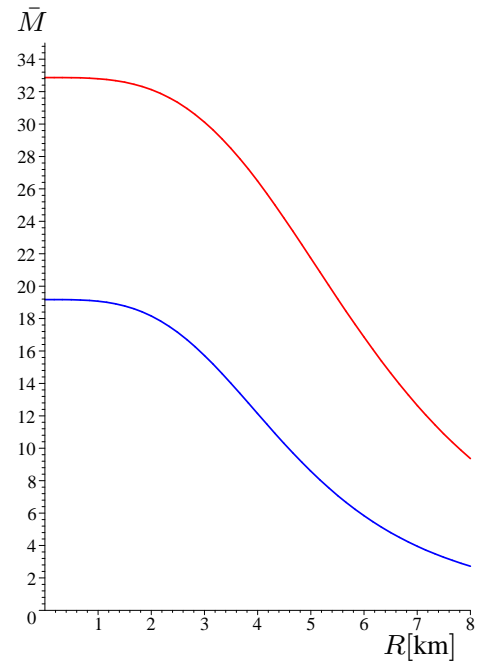

[DL]

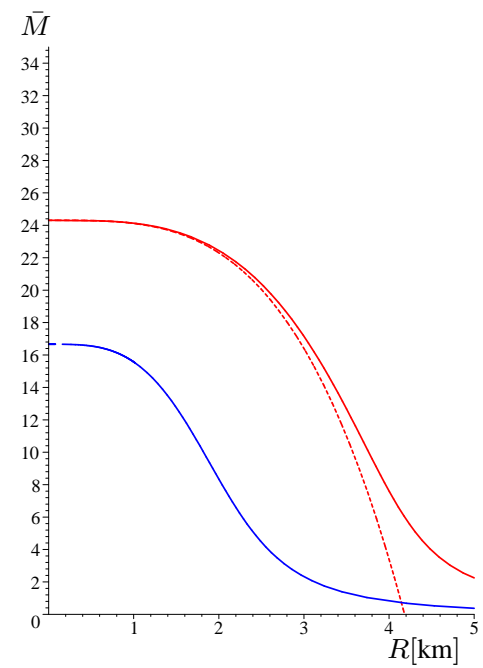

[UL]

Figure 1: Mean-load estimations for the downlink (DL) and uplink (UL), for the Hex model (upper curves) and PV model (lower curves). The dashed curve for the UL represents estimator for the Hex model with fully guaranteed coverage.

\subsection{Mean Load Estimations}

The mean-load estimations given by formulas (6.2) and (6.5), are presented on Figure 1. The upper curves correspond to the Hex model and lower curves to the PV model.

For the DL, the mean load with maximal power $\tilde{P}=\infty$ is about 38 and 22 for Hex and PV, respectively, and does not depend on $R$. On the plots we can recognize a region of (small) $R$ for which $\bar{M}$ is constant, where the power constraint can be ignored and the E-DPAFC can be replaced by DPAFC with $1-\pi$ on the right-hand-side of (4.1).

For the UL, the mean load with maximal power $\tilde{P}=\infty$ is about the same as load at $R=0$. We can also recognize a region of (small) $R$ for which $\bar{M}$ is constant, where the power constraint can be ignored and the UBC\&ULC can be replaced by UPAFC. The dashed curve for the UL presents mean load for Hex model with fully guaranteed coverage (formula (6.7), whereas the solid curve for this case represents the coverage guaranteed "in mean" (formula (6.5)).

\subsection{Maximal Load for given CPR}

We concentrate on Hex model. In order to estimate the maximal load at given CPR, we estimate per-cell probabilities of user rejection when applying E-DPAFC for the downlink and UBC\&ULC for the uplink. Figure 2 presents simulated (solid lines) and Gaussian-approximated (dashed lines) probabilities for various cell sizes. For the DL, we have from the right to the left: probability for DPAFC (which does not depend on $R$ ), then for E-DPAFC with $R=1,2,3,4,5$. For the UL, from 

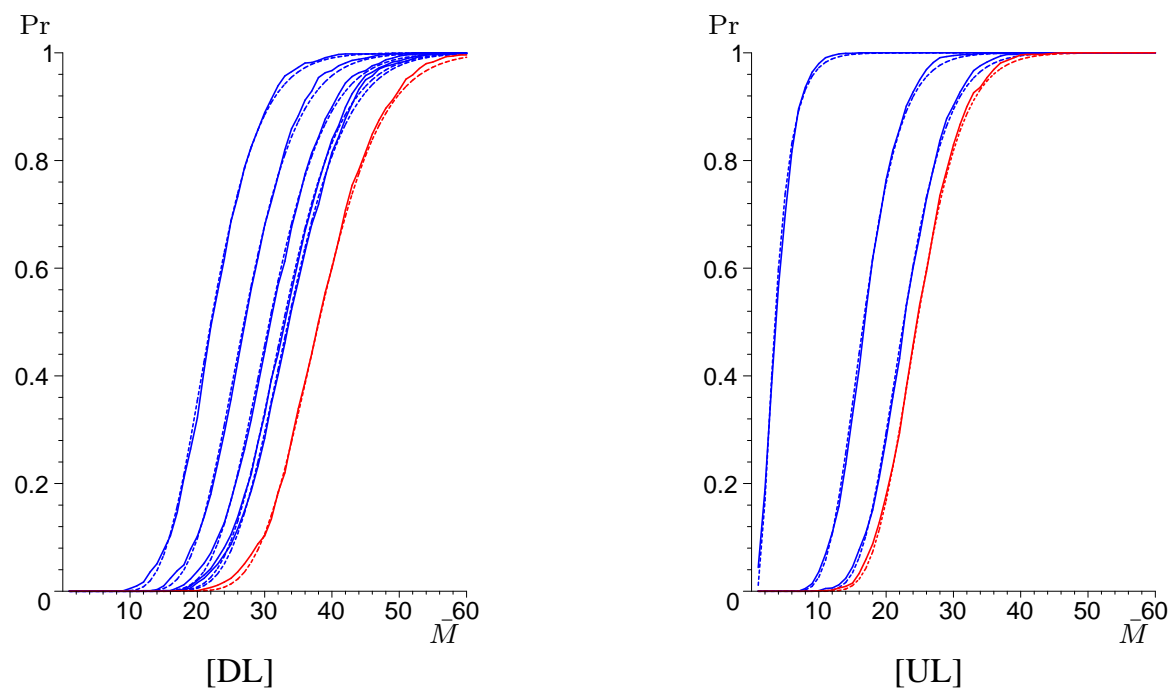

Figure 2: Probability of rejection in EDPAFC (DL) and in SE-UACP (UL) as function of $\bar{M}$ for various $R$. Simulated — solid line, Gaussian approximation — dashed line.

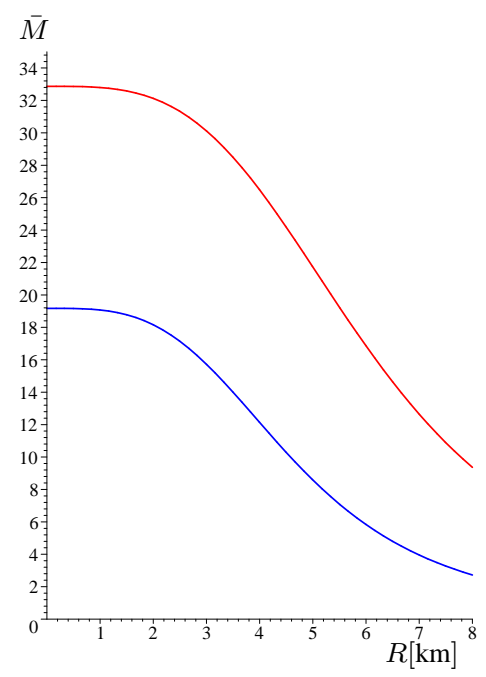

[DL]

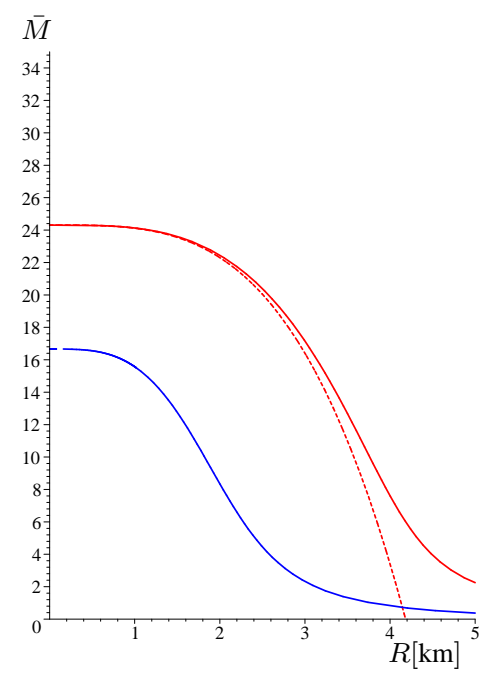

[UL]

Figure 3: Maximal load estimations in Hexagonal model at a given $\mathrm{CPR} \epsilon=$ $0.2,0.1,0.05,0.02,0.01$ (solid lines) for the downlink (DL) and uplink (UL). The dashed curves represent the values for the mean model. 
the right to the left we have: probability for UPAFC (which does not depend on $R$ and is about the same as this for SE-UACP for $R=1$ ) and then SE-UACP for $R=2,3,4$. We see that Gaussian approximation fits relatively well the simulated values. We can thus use these approximations to get maximal load estimations (6.14) and (6.15). Numerical results for $\epsilon=0.2,0.1,0.05,0.02,0.01$ are presented on Figure 3.

\section{Conclusion}

This paper builds scalable and decentralized admission /congestion control schemes. These algorithms take into account in an exact way the influence of the geometry on interferences as well as the existence of maximal power constraints.

Analytical methods for evaluating the global up and down-link maximal load of CDMA networks are given. Closed form approximations are built. Numerical studies show that these approximations are convenient and permit fast capacity evaluation of large UMTS networks.

The maximal load evaluation is based on the probability of non-feasible configurations. Another possible evaluation criterion is the blocking probability for voice users. We will try to build closed form expressions for the blocking probability in future studies.

\section{Appendix A: Summary of Mathematical Results}

This section collects mathematical formulas, proofs of statements, etc. Eventually it will be moved to a separate report or kept in an extended version of the paper.

\section{A.1 Proofs of feasibility conditions}

\section{A.1.1 Downlink}

The DPAFC condition (4.1) was derived in details in [1]. It is shown there that the feasibility problem of downlink power allocation without power constraints (i.e., condition 4.1.1i) is equivalent to existence of finite, non-negative solutions $\mathbf{H}=\left(H_{u}\right)$ of the following linear inequality

$$
(\mathbb{I}-\mathbb{A}) \mathbf{H} \geq \mathbf{b},
$$

were $\mathbb{A}=\left(a_{u v}\right), a_{u v}=\alpha_{u v} \sum_{m \in S_{u}} \xi_{\downarrow_{m}}^{u} l_{\downarrow_{m}}^{u} / l_{\downarrow_{m}}^{v}, \mathbf{b}=\left(b_{u}\right), b_{u}=P^{\prime} u+\sum_{m \in S_{u}} \xi_{\downarrow_{m}}^{u} N_{m}^{u} l_{\downarrow_{m}}^{u}$ and $\mathbb{I}$ is the respective identity matrix. The inequality (A.1) has solutions if and only if the spectral radius of $\mathbb{A}$ is less then 1. A sufficient condition for this proposed in [1], and saying that $\mathbb{A}$ has all linesums less than 1 (substochasticity of $\mathbb{A}$ ), is our DPAFC (4.1) condition. Condition E-DPAFC (4.2) is equivalent to saying that $(\mathbb{I}-\mathbb{A}) \tilde{\mathbf{P}} \geq \mathbf{b}$ where $\tilde{\mathbf{P}}=\left(\tilde{P}^{u}\right)$ that is obviously sufficient for existence of solutions of (A.1). Now, it is shown in [1] that if $\left(\tilde{P}^{u}\right)$ satisfy (A.1) then $P_{\downarrow_{m}}^{u}=\tilde{P}^{u} f_{\downarrow}^{u}$ for $m \in S_{u}$ are (minimal) solutions of the problem 4.1.1i. Obviously, under E-DPAFC (4.2) the power constraints 4.1.1ii are satisfied. 


\section{A.1.2 Uplink}

Denote $\mathbf{J}=\left(J_{u}\right), J_{u}=N^{u}+\sum_{v} \sum_{n \in S_{v}} P_{\uparrow}^{v}{ }_{n}^{v} l_{\uparrow}^{u}$ and let $\mathbb{B}=\left(b_{u v}\right), b_{u v}=\sum_{n \in S_{v}} \xi_{{ }_{\uparrow}}^{v} l_{\uparrow_{n}}^{v} / l_{\uparrow_{n}}^{u}$, $\mathbf{N}=\left(N^{u}\right)$. Note that feasibility of the uplink power control problem 4.1.1i with power constraints $4.1 .1 \mathrm{ii}$ is equivalent to the existence of non-negative finite solutions $\mathbf{J}=\left(J_{u}\right)$ of the inequality

$$
(\mathbb{I}-\mathbb{B}) \mathbf{J} \geq \mathbf{N}
$$

under constraints for all $u$

$$
J_{u} \xi_{\uparrow_{m}^{\prime}}^{u} l_{m}^{u} \leq \tilde{P}_{m}^{u} \quad \text { for all } m \in S_{u} .
$$

The condition UPAFC (4.3) says that the matrix $\mathbb{B}$ is substochastic (has all sums in columns) less than 1, and thus it is a sufficient condition for the existence of solutions of (A.2). The condition ULC (4.5) says that $J_{u}=N^{u} /\left(1-\theta_{u}\right)$ is a solution of (A.2) and UBC (4.4) guarantees (A.3). For the E-UPAFC, denote $\mathbb{C}=\left(c_{u v}\right), c_{u v}=\gamma_{u v} /\left(N^{v}+\gamma_{v}\right)$, and note that the condition (4.7) means that $\mathbb{B} \leq \mathbb{C}$ coordinate-wise. Thus the solution $J_{u}=N^{u}+\gamma_{u}$ of the inequality $(\mathbb{I}-\mathbb{C}) \mathbf{J} \geq \mathbf{N}$ is also a solution of (A.2). Moreover, condition (4.6) guarantees (A.3).

\section{A.2 Means and Probabilities}

We will consider probabilities and means calculated for two models, (Hex) and (PV) described in sections 6.1.1 and 6.1.2 with path-loss function given by (6.1). We will use notation $\stackrel{\text { Hex }}{=}$ and $\stackrel{P V}{=}$ to mark equalities that hold for the respective models; = denotes equality that holds for both models. Similar convention is adopted for other relations, as e.g., $\leq, \approx$. Moreover $l$ hs(number) denotes the left-hand-side of the formula with the given number. If it is not stated otherwise, all expectations are taken with respect to the so called Palm probability given there is a (typical) BS at the origin (see e.g., [15]).

\section{A.2.1 Means of the sums $\sum_{m \in S_{0}}[\ldots]$}

$$
\begin{aligned}
\mathrm{E}\left[\alpha_{0} \sum_{m \in S_{0}}{\xi_{\downarrow}^{\prime 0}}_{m}^{0}\right. & =\frac{\lambda_{M}}{\lambda_{B S}} \alpha_{0} \mathrm{E}\left[{\xi_{\downarrow}^{\prime}}^{0}\right]=\bar{M} \alpha \bar{\xi}_{\downarrow}^{\prime} \\
\mathrm{E}\left[\sum_{m \in S_{0}} N_{m}^{0}{\xi_{\downarrow}^{\prime}}_{m}^{0} l_{\downarrow}^{0}\right] & =\bar{M} \bar{N} \bar{\xi}_{\downarrow}^{\prime} L(R) \bar{g} \\
\mathrm{E}[\operatorname{lhs}(4.1)] & =\bar{M} \alpha \bar{\xi}_{\downarrow}^{\prime}(1+\bar{f})
\end{aligned}
$$

where $\bar{f}$ and $\bar{g}$ depend only on the propagation exponent $\eta$. For PV model, $\bar{f} \stackrel{\text { PV }}{=} 2 /(\eta-2), \bar{g} \stackrel{\text { PV }}{=}$ $\Gamma(1+\eta / 2)$, (see [1]). For Hex model, we suppose that base stations are placed on a regular hexagonal grid and we approximate cells with discs with the same area as the hexagons. It is easy to show that $\bar{g} \stackrel{\text { Hex }}{\approx} 1 /(1+\eta / 2)$. On the other hand numerical calculation of $\bar{f}$ shows that $\bar{f} \stackrel{\text { Hex }}{\approx} 1 /(\eta-2)$. 


\section{A.2.2 Variances of the sums $\sum_{m \in S_{0}}[\ldots]$}

Note that for the Hex model, the sum $\sum_{m \in S_{0}}\left[f_{m}\right]$ is a compound Poisson random variable and we have $\operatorname{Var}\left[\sum_{m \in S_{0}} f_{m}\right]=\bar{M} \mathrm{E}\left[f_{m}^{2}\right]$. The moments of the random variable $f_{m}$ are calculated numerically.

\section{A.2.3 Distribution and mean of $\sup _{m \in S_{0}}[\ldots]$}

Now we will study the sup in (4.4). Its distribution function can be expressed by the Laplace transform of the shot noise

$$
\operatorname{Pr}\left(\sup _{m \in S_{0}}\left(\xi_{\uparrow_{m}^{\prime}}^{0} l_{\uparrow}^{0} / \tilde{P}_{m}^{0}\right) \leq z\right)=\mathrm{E}\left[\exp \left[\sum_{m \in S_{0}} \ln \left(\mathbf{I}\left(\xi_{\uparrow}^{\prime 0} l_{\uparrow}^{0} / \tilde{P}_{m}^{0} \leq z\right)\right)\right]\right]
$$

and thus, for any model with Poisson arrivals

$$
\operatorname{Pr}\left(\sup _{m \in S_{0}}(\ldots) \leq z\right)=\mathrm{E}_{B S}\left[\exp \left(-\lambda_{M}\left|V^{0}\right| \operatorname{Pr}\left({\xi_{\uparrow}^{\prime 0}}_{\uparrow_{m}^{0}}^{0} / \tilde{P}_{m}^{0}>z\right)\right)\right]
$$

where $\left|V^{0}\right|$ is the area covered by BS 0 and $\mathrm{E}_{B S}[\ldots]$ means averaging over BS's configuration and concerns only the PV model. Consequently (by Jensen inequality for PV model)

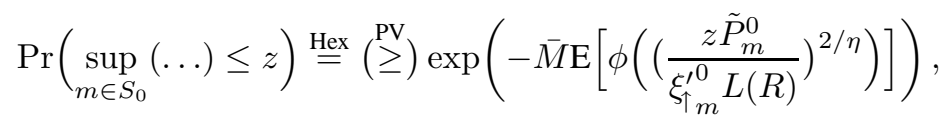

where the function $\phi()$ depends on the model and

$$
\begin{aligned}
& \phi(r) \stackrel{\text { PV }}{=} \quad e^{-r} \quad \text { for } r \geq 0 \\
& \phi(r) \stackrel{\text { Hex }}{=} \begin{cases}1-r & \text { for } 0 \leq r \leq \frac{\pi}{2 \sqrt{3}} \\
1-r & -\sqrt{\frac{6 \sqrt{3} r}{\pi}-3}+\frac{6 r}{\pi} \arccos \left(\frac{\sqrt{\pi \sqrt{3}}}{\sqrt{6} r}\right) \\
& \text { for } \frac{\pi}{2 \sqrt{3}} \leq r \leq \frac{2 \pi}{3 \sqrt{3}}\end{cases}
\end{aligned}
$$

Note that $\phi(r) \stackrel{\text { Hex }}{\geq} \max (0,1-r)$ and in sequel, we will use the approximation $\phi(r) \stackrel{\text { Hex }}{\approx} \max (0,1-r)$. For the expectation, we have thus

$$
\mathrm{E}\left[\sup _{m \in S_{0}}(\ldots)\right] \stackrel{\text { Hex }}{=}(\stackrel{\mathrm{PV}}{\leq}) \int_{0}^{\infty} 1-\exp \left[-\bar{M} \mathrm{E}\left[\phi\left(\left(\frac{z \tilde{P}_{m}^{0}}{\xi_{\uparrow_{m}^{\prime}}^{0} L(R)}\right)^{2 / \eta}\right)\right]\right] d z
$$

Now, developing in Taylor series with respect to $\bar{M}$, we have for the Hex model

$$
\int_{0}^{\infty} 1-\exp [-\bar{M} \mathrm{E}[\phi(\ldots)]] d z \stackrel{\mathrm{Hex}}{\approx} L(R)\left(\mathrm{E}\left[\frac{\tilde{P}_{m}^{0}}{\xi_{\uparrow_{m}^{\prime}}^{0}}\right]^{2 / \eta}\right)^{-\eta / 2} \sum_{n=1}^{\infty} \frac{(-1)^{n+1} \bar{M}^{n} \eta \Gamma(\eta / 2)}{2 \Gamma(\eta / 2+n+1)} .
$$

$\operatorname{RR} n^{\circ} 4954$ 
Similarly for PV model

$\int_{0}^{\infty} 1-\exp [-\bar{M} \mathrm{E}[\phi(\ldots)]] d z \stackrel{\mathrm{PV}}{=} L(R) \frac{\eta}{2} \Gamma\left(\frac{\eta}{2}\right) \sum_{n=1}^{\infty} \frac{(-1)^{n+1} \bar{M}^{n}}{n !} \mathrm{E}\left[\left(\sum_{m=1}^{n}\left(\frac{\tilde{P}_{m}^{0}}{\xi_{\uparrow}^{\prime 0}}\right)^{2 / \eta}\right)^{-\eta / 2}\right]$.

Concluding, note that in the case of constant $\xi_{\uparrow}^{\prime u} / \tilde{P}_{m}^{u} \equiv \bar{\xi}_{\uparrow}^{\prime} / \bar{P}_{\uparrow}$ we have

$$
\left.\mathrm{E}\left[\sup _{m \in S_{0}}\left(\xi_{\uparrow}^{\prime 0} l_{\uparrow}^{0} / \tilde{P}_{m}^{0}\right)\right] \stackrel{\text { Hex }}{\stackrel{\mathrm{PV}}{\leq}}\right) L(R) \bar{\xi}_{\uparrow}^{\prime} \bar{h}(\bar{M}) / \tilde{P}
$$

where

$$
\begin{aligned}
& \bar{h}(s) \stackrel{\mathrm{Hex}}{\approx} \int_{0}^{1} 1-e^{-s\left(1-z^{2 / \eta}\right)} d z=\sum_{n=1}^{\infty} \frac{(-1)^{n+1} s^{n} \eta \Gamma(\eta / 2)}{2 \Gamma(\eta / 2+n+1)} \\
& \bar{h}(s) \stackrel{\mathrm{PV}}{=} \int_{0}^{\infty} 1-e^{-s e^{-z^{2 / \eta}}} d z=\frac{\eta}{2} \Gamma\left(\frac{\eta}{2}\right) \sum_{n=1}^{\infty} \frac{(-1)^{n+1} s^{n}}{n ! n^{\eta / 2}}
\end{aligned}
$$

\section{References}

[1] F. Baccelli, B. Bł aszczyszyn, and F. Tournois, "Downlink admission/congestion control and maximal load in large CDMA networks," in Proceedings of IEEE INFOCOM'03, San Francisco, April 2003, preprint.

[2] R. Nettleton and H. Alavi, "Power control for spread spectrum cellular mobile radio system," Proc. IEEE Trans. Veh. Technol. Conf., pp. 242-246, 1983.

[3] K. S. Gilhousen, I. M. Jacobs, R. Padovani, A. J. Viterbi, L. A. Weaver Jr., and C. E. Wheatley III, "On the capacity of a cellular CDMA system," IEEE Trans. Veh. Technol., vol. 40, pp. 303-312, 1991.

[4] J. Zander, "Performance of optimum transmitter power control in cellular radio systems," IEEE Trans. Veh. Technol., vol. 41, pp. 57-62, 1992.

[5] _ "Distributed co-channel interference control in cellular radio systems," IEEE Trans. Veh. Technol., vol. 41, pp. 305-311, 1992.

[6] G. Foschini and Z. Miljanic, "A simple distributed autonomous power control algorithm and its convergence," IEEE Trans. Veh. Technol., vol. 40, pp. 641-646, 1991.

[7] S. Hanly, "Capacity in a two cell spread spectrum network," Thirtieth Annual Allerton Conf. Commun., Control and Computing, vol. IL, pp. 426-435, 1992.

[8] _— "Congestion measures in DS-CDMA networks," IEEE Trans. Commun., vol. 47, pp. 426-437, 1999. 
[9] C. Zhu and M. Corson, "A distributed channel probing for wireless networks," Proc. of IEEE Infocom 2001, pp. 403-411, 2001.

[10] S. Hanly, "Capacity and power control in spread spectrum macrodiversity radio networks," IEEE Trans. Commun., vol. 44, pp. 247-256, 1996.

[11] T. N. Jaana Laiho, Achim Wacker, Radio Network Planning and Optimisation for UMTS, J. Wiley and sons, Eds., 2002.

[12] S. Hanly, "Congestion measures in DS-CDMA networks," IEEE Trans. on Comm., vol. 47, 3 1999.

[13] J. Zander and M. Frodigh, "Comment on performance of optimum transmitter power control in cellular radio systems," IEEE Trans. Vehic. Technol., vol. 43, pp. 636-636, August 1994.

[14] B. Radunović and J. L. Boudec, "A unified framework for max-min and min-max fairness with applications," Proc. of Allerton'02, 2002.

[15] D. Stoyan, W. Kendall, and J. Mecke, Stochastic Geometry and its Applications. John Wiley \& Sons, Chichester, 1995. 
Unité de recherche INRIA Rocquencourt Domaine de Voluceau - Rocquencourt - BP 105 - 78153 Le Chesnay Cedex (France)

Unité de recherche INRIA Lorraine : LORIA, Technopôle de Nancy-Brabois - Campus scientifi que 615, rue du Jardin Botanique - BP 101 - 54602 Villers-lès-Nancy Cedex (France)

Unité de recherche INRIA Rennes : IRISA, Campus universitaire de Beaulieu - 35042 Rennes Cedex (France)

Unité de recherche INRIA Rhône-Alpes : 655, avenue de l'Europe - 38330 Montbonnot-St-Martin (France)

Unité de recherche INRIA Sophia Antipolis : 2004, route des Lucioles - BP 93 - 06902 Sophia Antipolis Cedex (France) 